\title{
Another Great Imitator: Allergic Contact Dermatitis Differential Diagnosis, Clues to Diagnosis, Histopathology, and Treatment
}

Jessica K. So, $M D^{1}$

Ashley Hamstra, $M D^{2}$

Antoanella Calame, $M D^{3,4}$

Carsten R. Hamann, $M D^{5}$

Sharon E. Jacob, $M D^{6, *}$

\author{
Address \\ ${ }^{1}$ Division of Dermatology, Sharp Rees-Stealy Medical Group, La Mesa, CA, USA \\ ${ }^{2}$ Department of Dermatology, Medical University of South Carolina, Charleston, \\ SC, USA \\ ${ }^{3}$ Department of Dermatology, UC San Diego, La Jolla, CA, USA \\ ${ }^{4}$ Compass Dermatopathology, La Jolla, CA, USA \\ ${ }^{5}$ Department of Medicine, Loma Linda University, Loma Linda, CA, USA \\ ${ }^{*}, 6$ Department of Dermatology, Loma Linda University, Faculty Medical Offices, \\ 11370, Anderson Street, Suite 2600, Loma Linda, CA, 92354, USA \\ Email: sjacob@contactderm.net
}

Published online: 16 0ctober 2015

(C) Springer International Publishing AG 2015

This article is part of the Topical Collection on Contact Dermatitis

Keywords Dermatitis - Allergic contact dermatitis - Atopic dermatitis

\section{Opinion statement}

Allergic contact dermatitis (ACD) is a type IV (delayed) hypersensitivity reaction that has a wide spectrum of presentations that often imitate or overlap with other cutaneous eruptions. Differential diagnoses to consider include infections, skin lymphoma-malignancies, inflammatory dermatoses, nutritional deficiencies, and mechanical causes of tissue damage. We discuss clues to the diagnosis of $A C D$, such as pruritus, localization to the area of skin contact with the allergen, recurrence with repeat exposures, and supportive skin biopsy histology.

Epicutaneous patch testing remains the gold standard for diagnosing ACD. Definitive treatment is contact allergen avoidance and, when indicated, dietary restriction. With these measures, most patients will improve clinically. In cases 
where avoidance measures and interim topical therapies fail, ultraviolet light therapy or systemic immunosuppression may be considered.

\section{Key points}

1. Allergic contact dermatitis (ACD) is a type IV (delayed) hypersensitivity reaction with a range of clinical presentations.

2. Mimickers of ACD include infections, skin lymphoma-malignancies, inflammatory dermatoses, nutritional deficiencies, and mechanical causes of tissue damage.

3. Allergen avoidance, which might include dietary restriction, is the definitive treatment for ACD.

\section{Introduction}

Allergic contact dermatitis (ACD) is a cutaneous manifestation of delayed-type hypersensitivity (type IV) reactions, usually manifesting as pruritic eczematoid changes of the skin. Barrier defects further enable allergen entry, subsequent processing, and presentation of the allergen to naïve $\mathrm{T}$ cells by dendritic cells. This process is defined as "sensitization" and results in the clonal expansion of memory $\mathrm{T}$ cells. After repeated exposure to a particular allergen, the second "re-activation" phase of ACD, named "elicitation," occurs. Depending on the level of sensitization and frequency of repeat elicitations, some patients develop intermittent dermatitis while others develop a persistent dermatitis. The persistent dermatitis may expand from a localized presentation to a generalized dermatitis. In addition, a localized reaction may be diffuse or patchy within the exposed area, making identification challenging.

For diagnosis and definitive treatment $\mathrm{ACD}$ must be considered within a broad differential diagnosis, but the differential can be narrowed with careful history taking and clues found on physical examination. Herein, we discuss a differential diagnosis of $\mathrm{ACD}$, clues to making the diagnosis, and finally a treatment algorithm for patients with disease refractory to avoidance measures.

\section{Epidemiology}

While ACD is known to affect a large percentage of the population, the exact prevalence in the general population is unknown. Estimations of contact sensitization prevalence have been calculated based on patch-tested populations with and without chronic dermatitis. In 2007, the estimated median prevalence of contact allergy in the general population was calculated at $21.2 \%$, with a weighted average prevalence of $19.5 \%$ [1]. One group of patients that may not be included in these estimates includes those with acute dermatitis, such as with toxicodendron (poison ivy). These patients less commonly present for dermatologic diagnostic evaluation and are more likely to either self-treat or present to the primary care practitioner. The pediatric population is likely under-diagnosed, as the Food and Drug Administration (FDA) has not yet approved patch testing in kids. Also, in patients with concomitant diagnoses, such as atopic dermatitis, the secondary diagnosis of ACD may remain undiagnosed.

Although differences in presentation and allergens follow exposure patterns, ACD affects a large percentage of the population, young and old, all races, and both genders. Populations at higher risk for ACD include those with occupational exposure, such as hairdressers, veterinarians, and athletes. This risk is heightened 


\section{Presentation}

in individuals with underlying atopy or other chronic skin barrier defects.

The eruption presentation is found on a spectrum of acute, subacute, or chronic. Poison oak is a prototypical example of acute cases, which displays microvesiculation, edema, and erythema. While chronic cases, such as those caused by personal hygiene products, tend to be more eczematous and less exudative in morphology, ACD can imitate a wide spectrum of cutaneous eruptions, from bullae formation mimicking bullous tinea to eczematous eruptions resembling irritant contact dermatitis, atopic dermatitis, asteatotic eczema, stasis dermatitis, and seborrheic dermatitis.

\section{Diagnosis}

The epicutaneous patch test remains the gold standard for diagnosing ACD. When positive patch test reactions are elicited, the potential relevance of the identified allergens to the dermatitis must be assessed. Exposure history to allergens, as well as correlation of relapses with exposures and distribution, should be noted.

When the diagnosis is in doubt and patch testing does not identify the relevant allergens or allergen avoidance fails to clear the eruption, skin biopsy for histopathologic examination may be helpful in distinguishing ACD from other conditions. Typically, in the very early stages of ACD, histology of lesional skin is characterized by spongiosis, usually most pronounced in the lower epidermis. Later, spongiotic vesicles may form at various levels in the epidermis. Infiltration of lymphocytes, Langerhans cells, and macrophages is seen in the upper dermis around superficial vessels. Eosinophils are frequently seen but their absence does not exclude ACD. Eosinophilic exocytosis is common in ACD. Chronic lesions reveal less spongiosis with more prominent epidermal hyperplasia, scale crust, and mild papillary dermal fibrosis. Hypergranulosis is often a feature in lesions that are rubbed. Spongiosis and vesiculation are much less common in chronic lesions [2]. A Periodic Acid-Schiff (PAS) stain can help rule out a dermatophyte infection.

\section{Differential diagnosis}

The differential diagnosis of ACD includes many dermatitides, such as atopic dermatitis, asteatotic eczema (eczema craquele), dyshidrotic eczema, erythrodermas, lichen planus, perioral dermatitis, psoriasis, rosacea, stasis dermatitis, seborrheic dermatitis and others.

The ACD reaction is usually localized to the area of skin contact with the allergen but may be diffuse or patchy within the exposed area. In either case, meticulous inspection of the distribution of an eruption is critical to diagnosis. For example, localization of the eruption to intertriginous areas should bring consideration of Hailey-Hailey, erythrasma, and candida. Groin ACD must be distinguished from extramammary Paget's disease, inverse psoriasis, and inverse lichen planus. ACD of the face, particularly around the mouth, may mimic nutritional deficiencies, periorificial dermatitis, or rosacea. 
Photodistribution of an eruption can also be a valuable clue to the diagnosis as it often suggests an element of photosensitivity. This can be seen in polymorphous light eruption or an underlying autoimmune connective tissue disease, such as cutaneous lupus erythematosus. The cutaneous manifestations of dermatomyositis often involve skin overlying the extensor surface of joints, the shoulders, face, and anterior chest. There is often a component of poikiloderma. These patients may present with or without associated muscle symptoms.

However, the distinction between photodistributed and exposed area dermatitis (airborne dermatitis) can be a very difficult one to make [3]. Exposed area dermatitis involves the same areas usually spared in photodistributed dermatitis, such as behind the ears (Wilkinson's triangle), nasolabial folds, and under the chin. ACD to personal hygiene products (cosmetics, fragrances, and lotions) often mimic photosensitivity as they also affect the face, neck, and arms.

Papular eruptions may resemble folliculitis or Grover's disease. Common skin infections, including dermatophytes (tinea), scabies, and bacterial or pityrosporum folliculitis, should also be considered and treated. Widespread contact with an allergen or autosensitization may be confused for other causes of erythroderma, such as Sézary syndrome or pustular psoriasis. Importantly, a high suspicion must be maintained for malignant neoplasms such as mycosis fungoides (cutaneous T cell lymphoma) or extramammary Paget's disease, as well as paraneoplastic disorders including acquired ichthyosis.

Any history involving possible mechanical trauma to the skin may offer a clue to an irritant contact dermatitis (ICD) or intertrigo. A common example of ICD is chronic hand dermatitis. It is typically symmetrically accentuated on the dorsal hands from just proximal to the metacarpophalangeal joint down to the fingertips (apron sign). This is related to hand washing technique. A history of frequent hand washing and this cutaneous finding would suggest a primarily ICD-induced dermatitis. Protein contact dermatitis is also often on the hand but classically occurs within minutes of exposure. Additionally, recent medication changes (including supplements and other over the counter agents) should raise consideration of a drug eruption.

Underlying medical conditions such as bowel disorders or psychiatric conditions may predispose to nutritional deficiencies, which can cause ACD mimickers. A salient example would be pellagra (niacin deficiency), which can induce a photosensitive dermatitis (Casal's necklace). Biotin, zinc, and essential fatty acid deficiencies present differently and can be in the differential for diaper dermatitis in infants. They present with periorificial pustules and bullae that evolve into scaly and crusted erosions. ACD of the diaper area is often secondary to preservatives in topical preparations. Diaper dermatitis is often multifactorial with ICD frequently playing a role.

\section{Prognosis}

Definitive remission of ACD requires the correct diagnosis and avoidance of causative allergens. In addition, barrier maintenance and repair should be addressed for complete clearance. With these measures, the prognosis is excellent. However, patients may have different "thresholds" for reactivity, whereby 


\section{Treatment}

a minute amount of allergen may initiate a significant clinical response in more sensitive patients. These patients must observe strict adherence to avoidance measures to remit the dermatitis and symptoms.

An algorithmic approach to the management of dermatitis in patients with clinically relevant positive patch tests can be found in Fig. 1, and a comparative review on treatment indications for the differential diagnosis is developed in Table 1.

Unfortunately, not all patients with positive patch test results will clear with avoidance recommendations. There are several possible reasons for recalcitrant dermatitis: the positive results may not have been relevant to the current dermatitis, the clinically relevant allergen was not captured on the patch test due to false negativity or due to not being a tested allergen, patient education/ counseling and comprehension for complete allergen avoidance may have been incomplete, and other routes of allergen exposure may have not been identified; in some cases, complete avoidance is not possible. When avoidance fails,
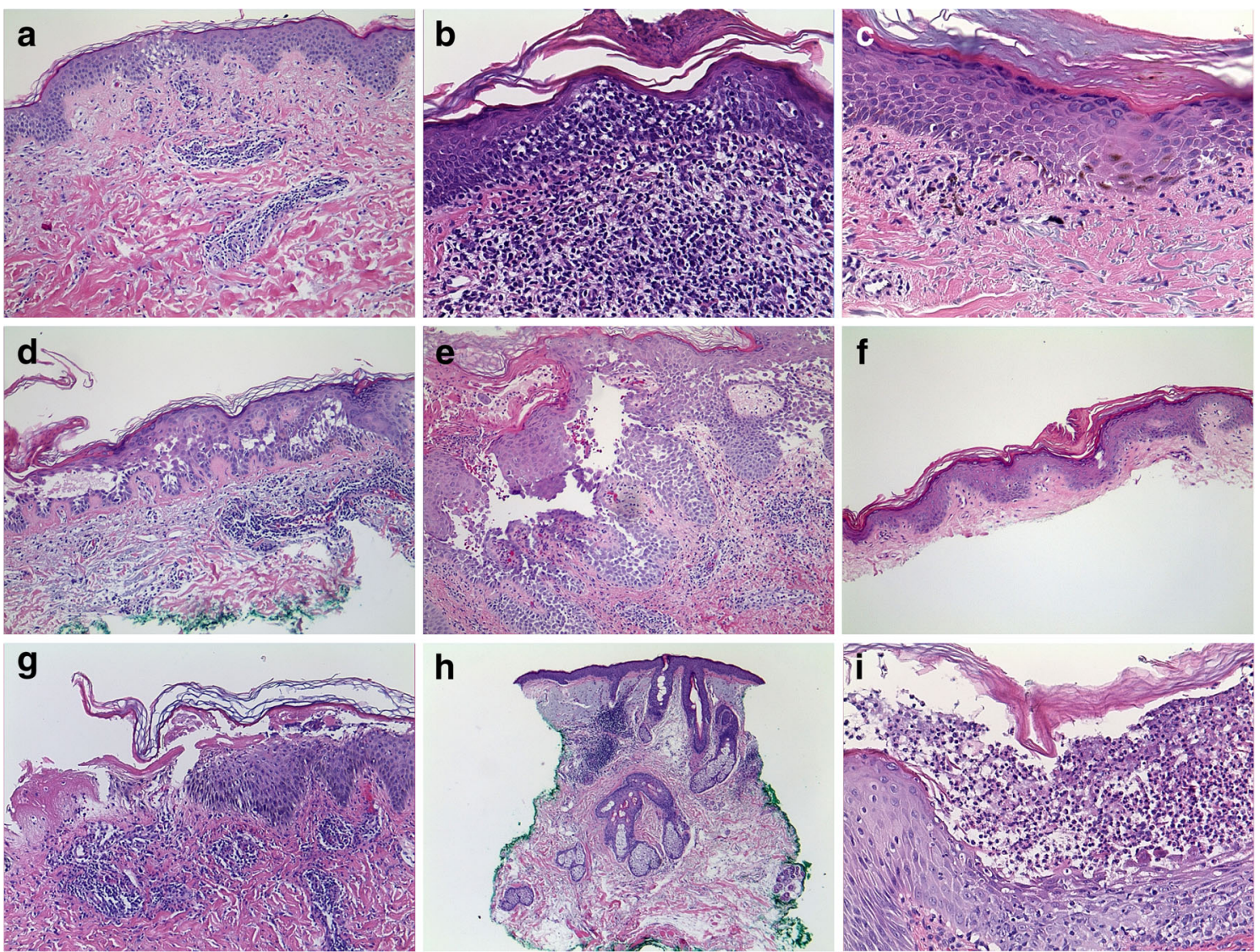

Fig. 1. a Allergic contact dermatitis at 10x. b Cutaneous T cell lymphoma at 20x. c Dermatomyositis at 20x. d Grover's disease at 10x. e Hailey-Haley disease at 4x. $\mathbf{f}$ Intertrigo at 10x. $\mathbf{g}$ Irritant contact dermatitis at 10x. $\mathbf{h}$ Perioral dermatitis at 10x. $\mathbf{i}$ Tinea corporis at20x. 


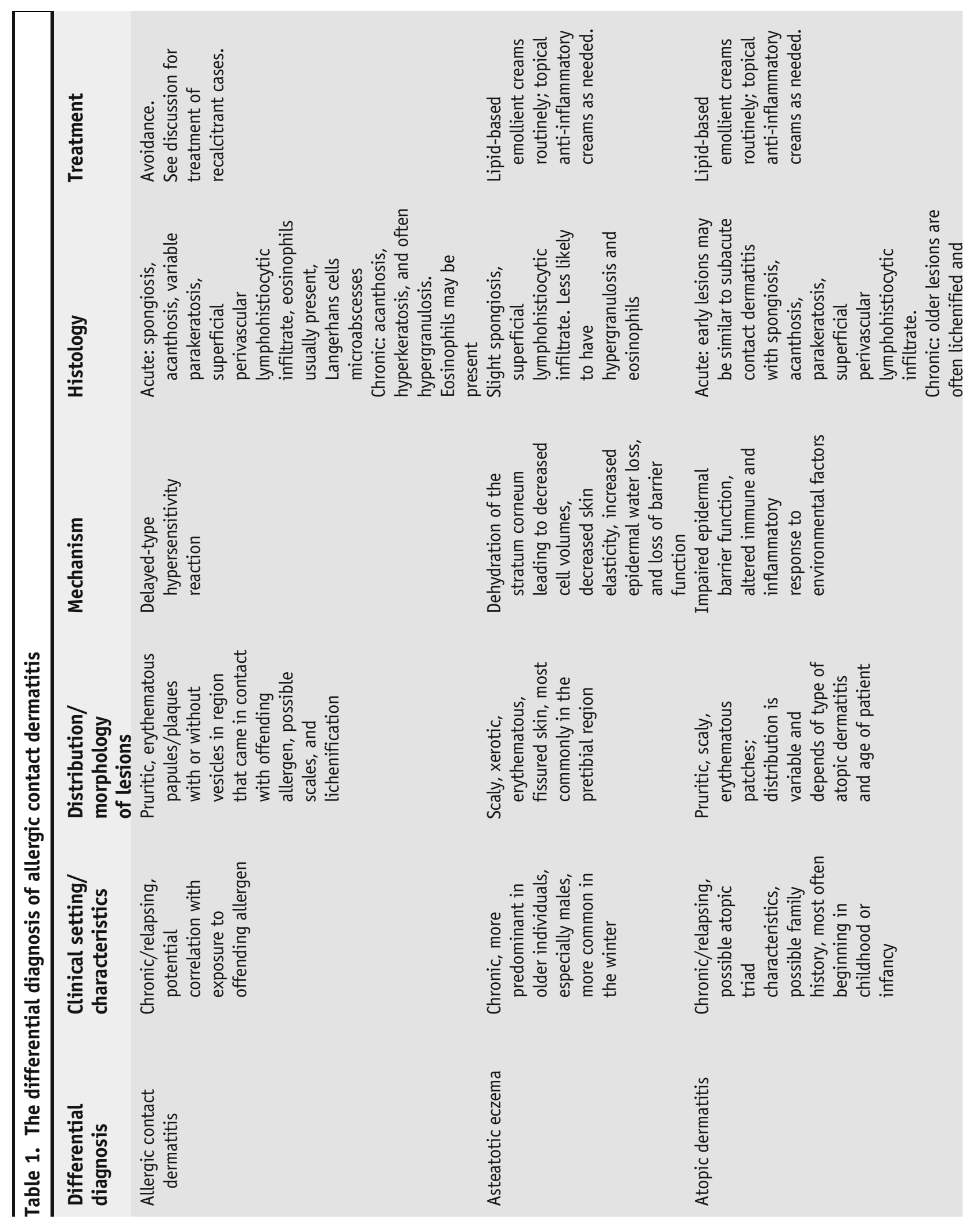




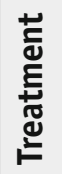
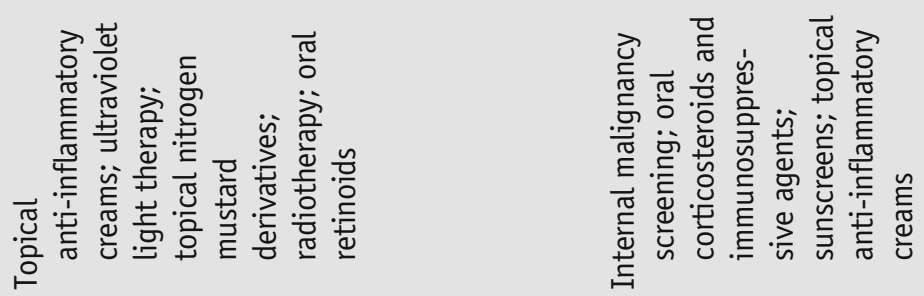

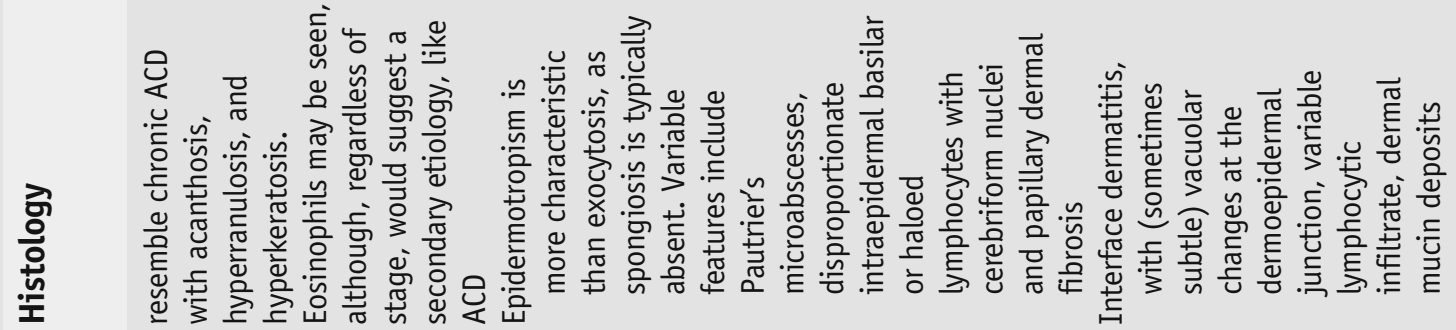

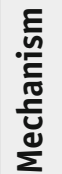
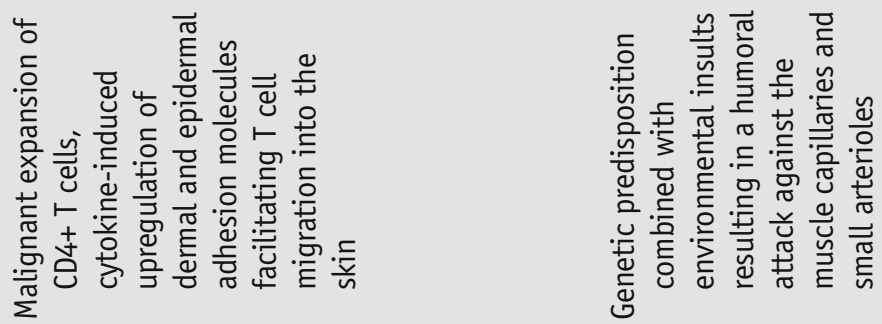

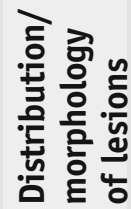

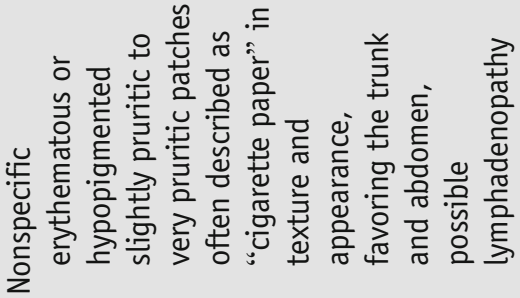

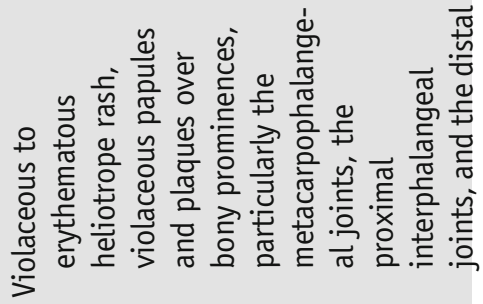

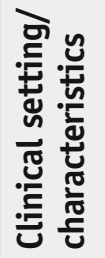

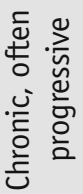
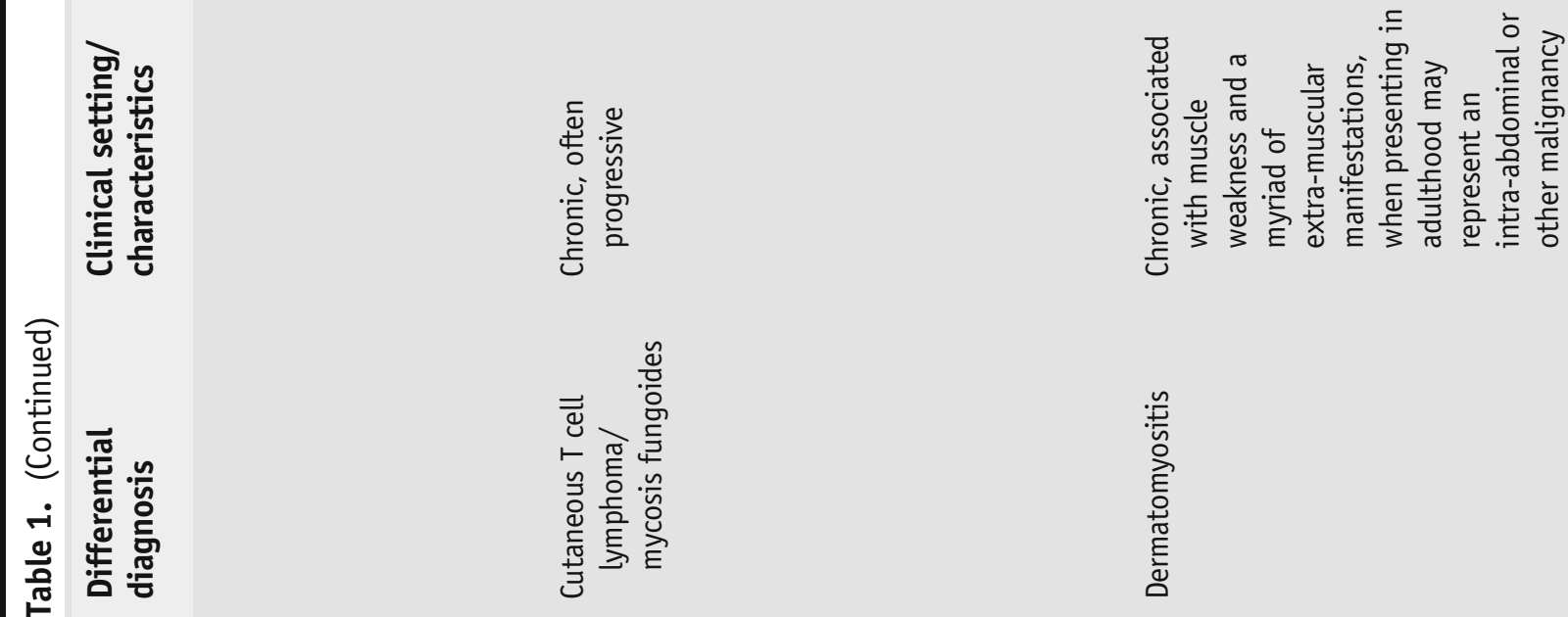

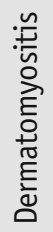



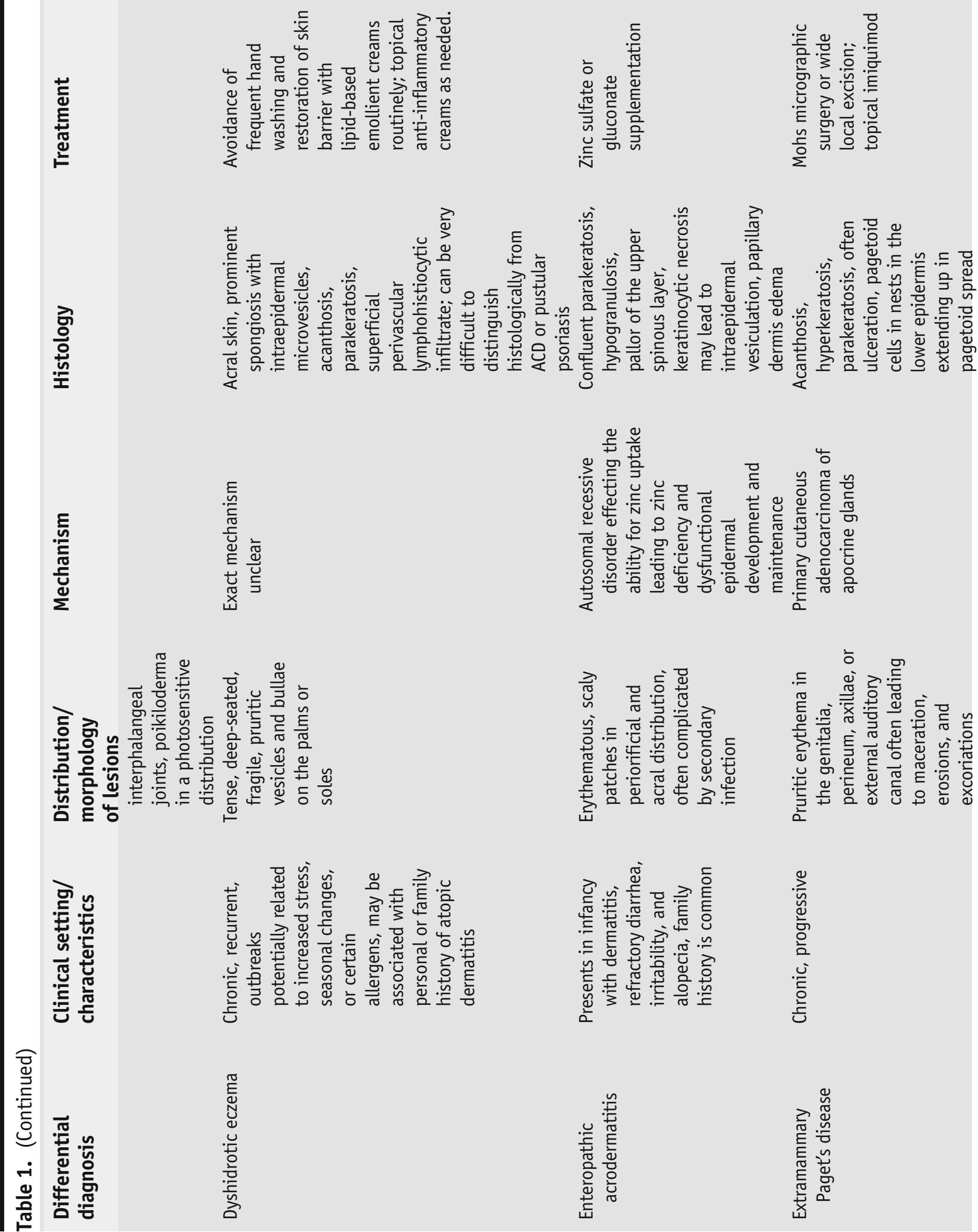


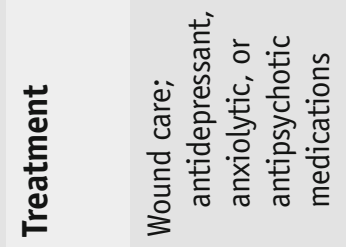
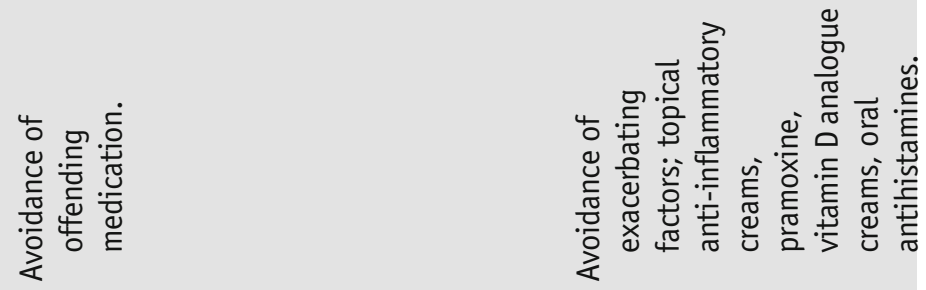

竞

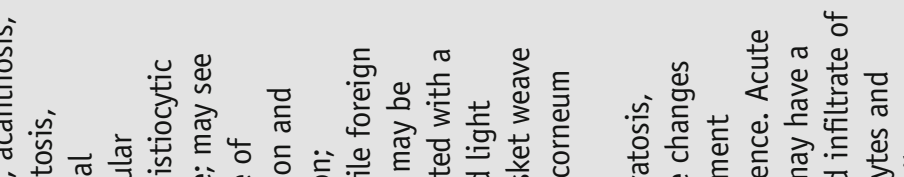

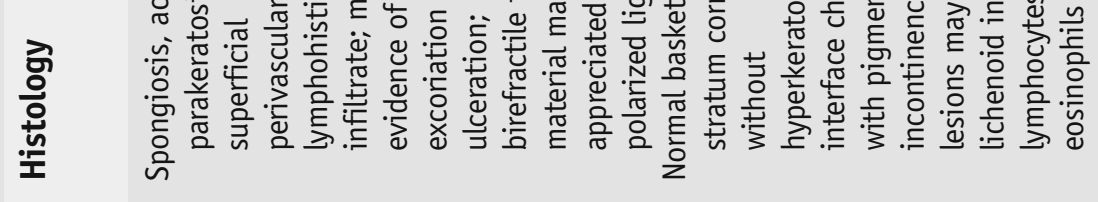

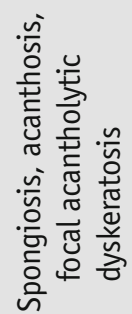

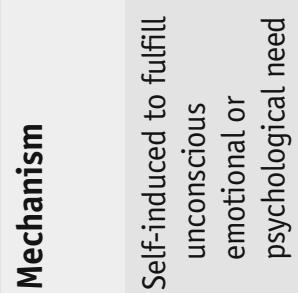
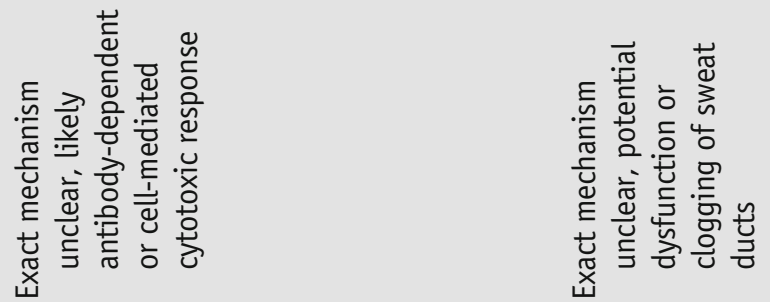

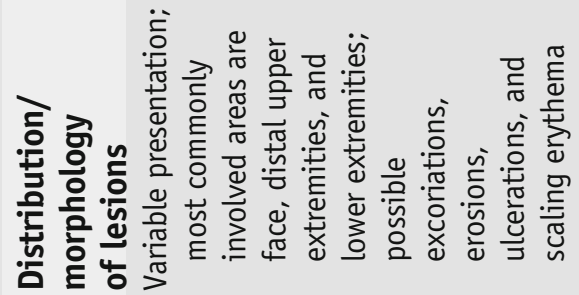
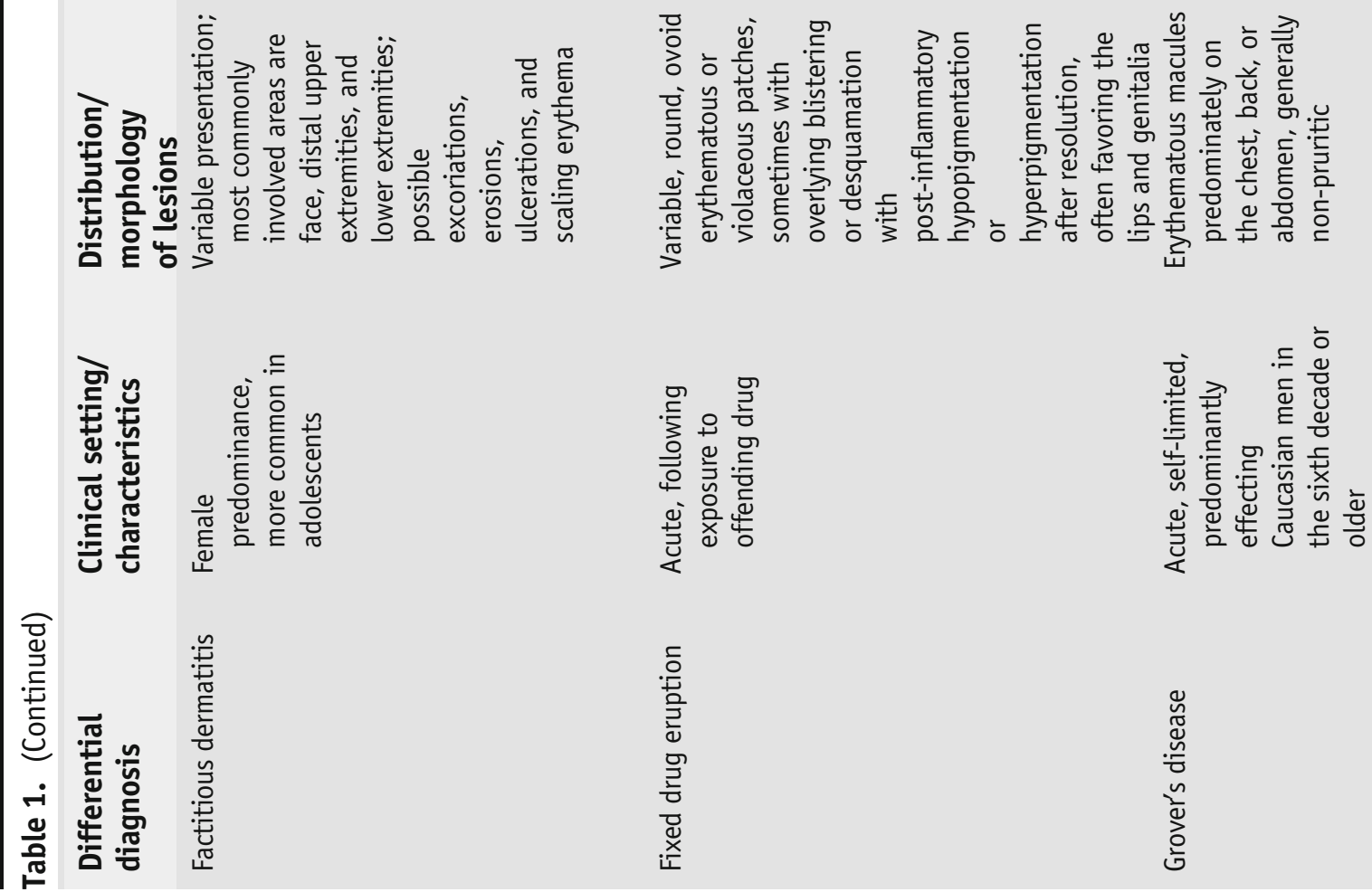

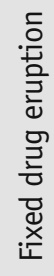

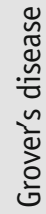




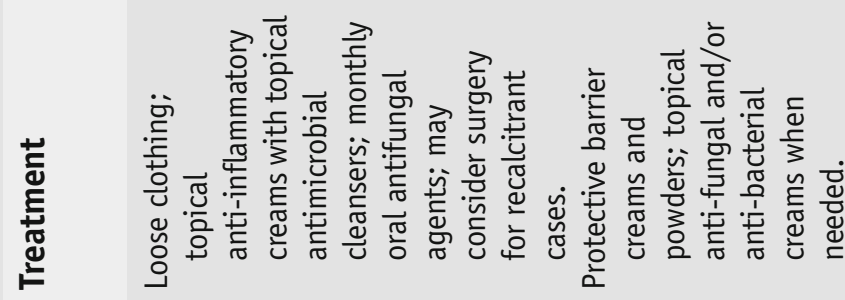
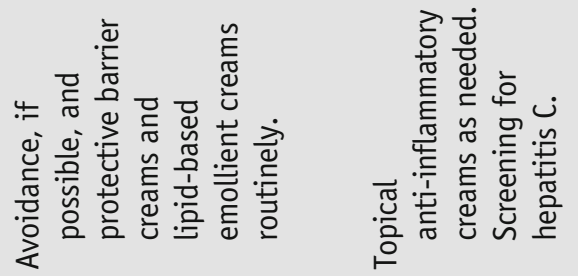

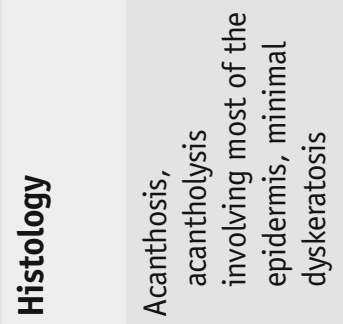
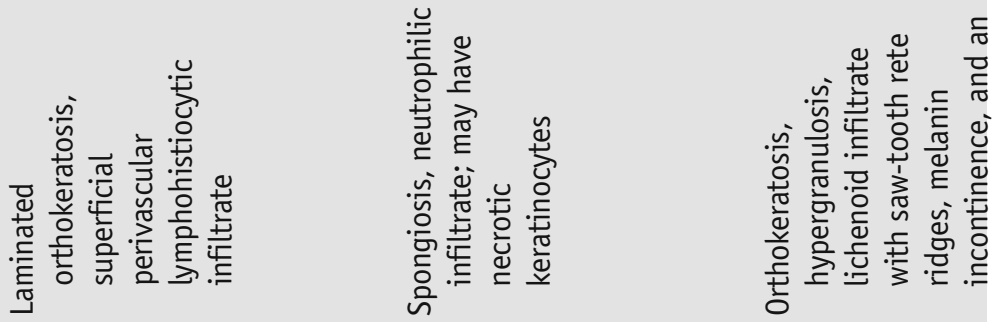

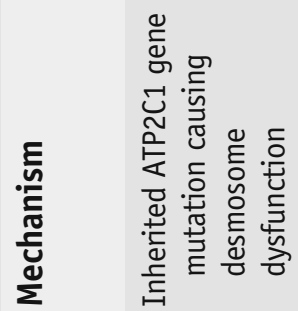

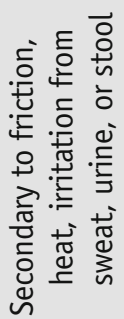

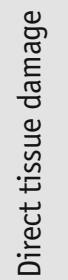

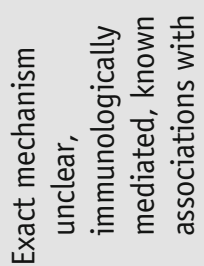

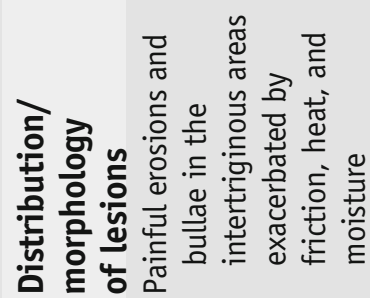
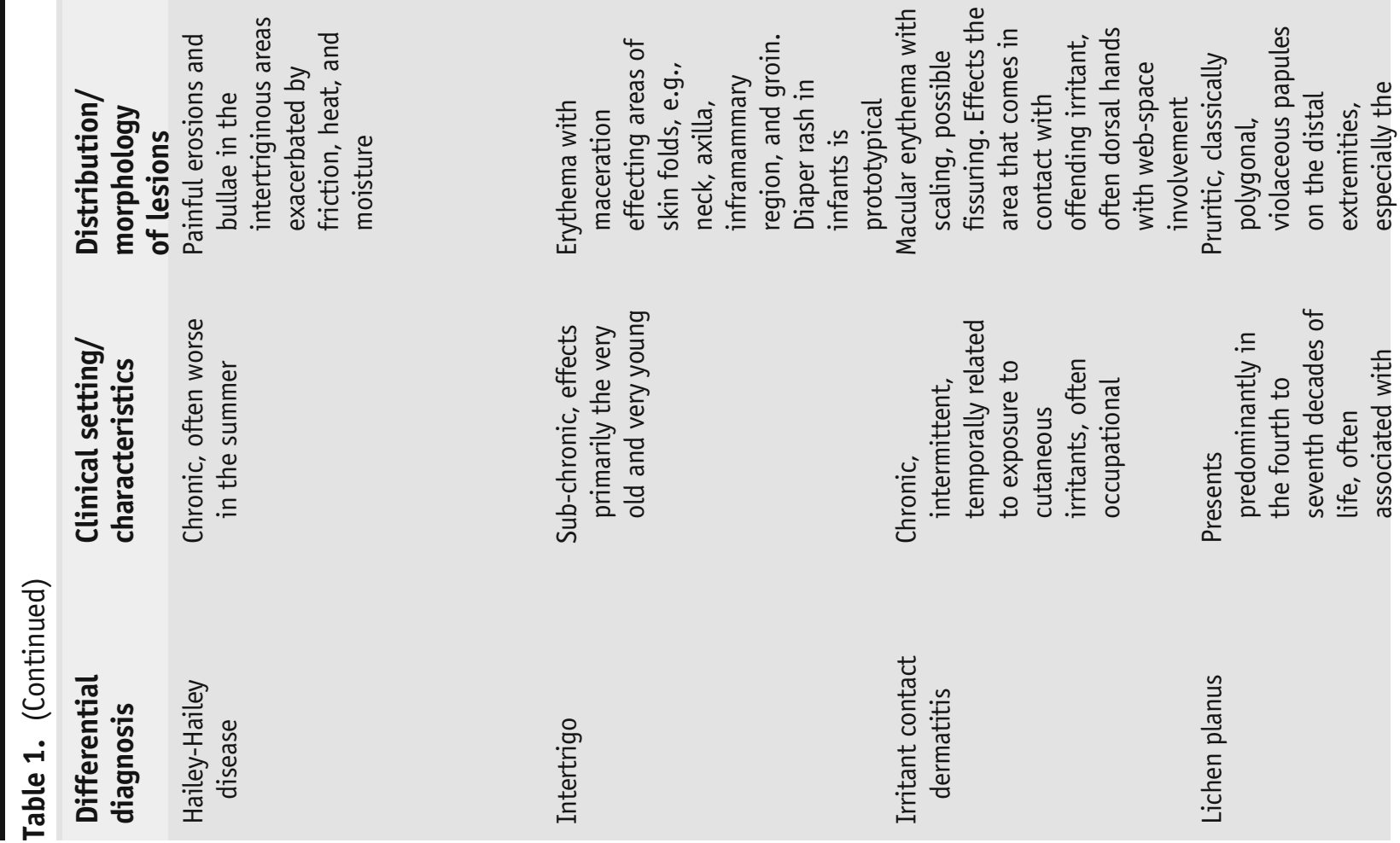

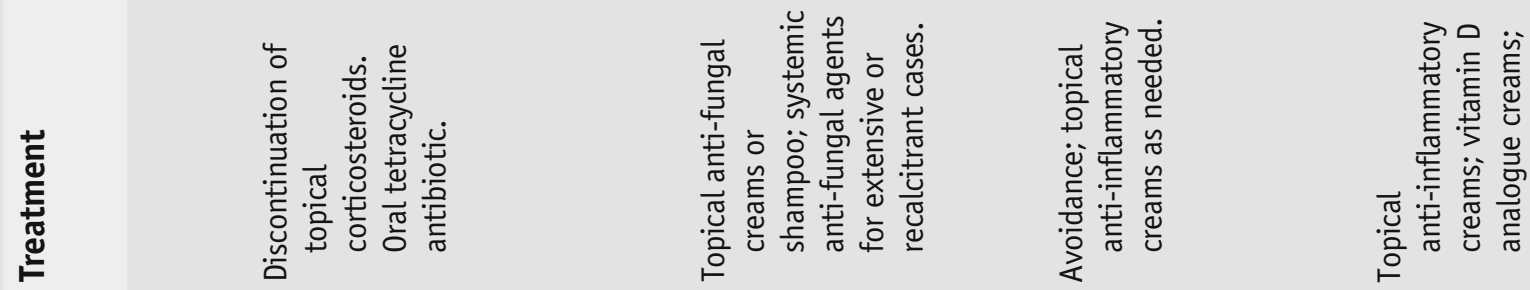

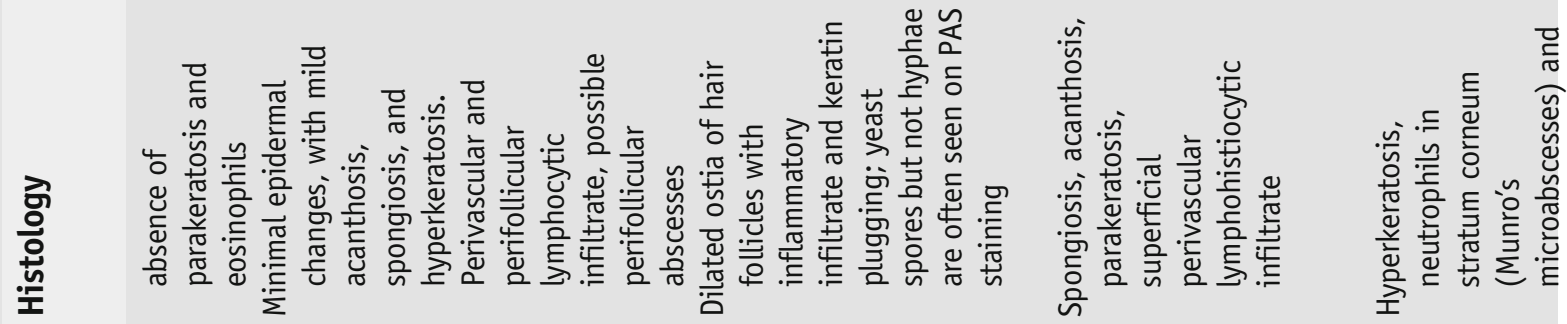
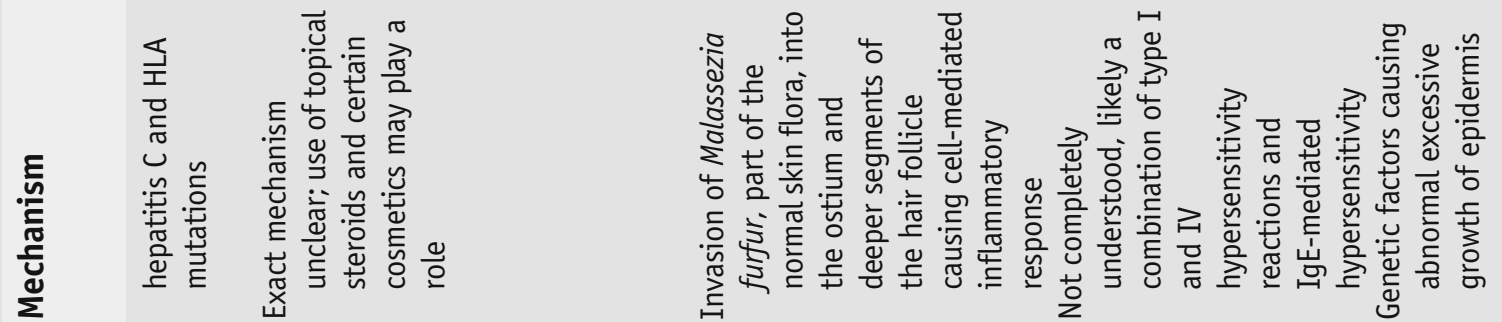

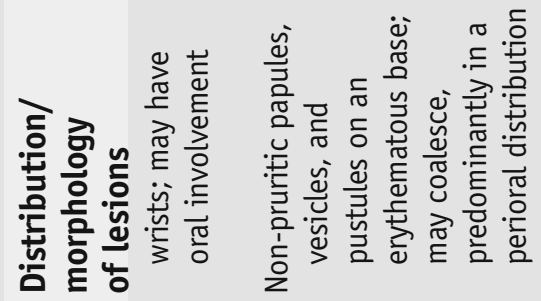
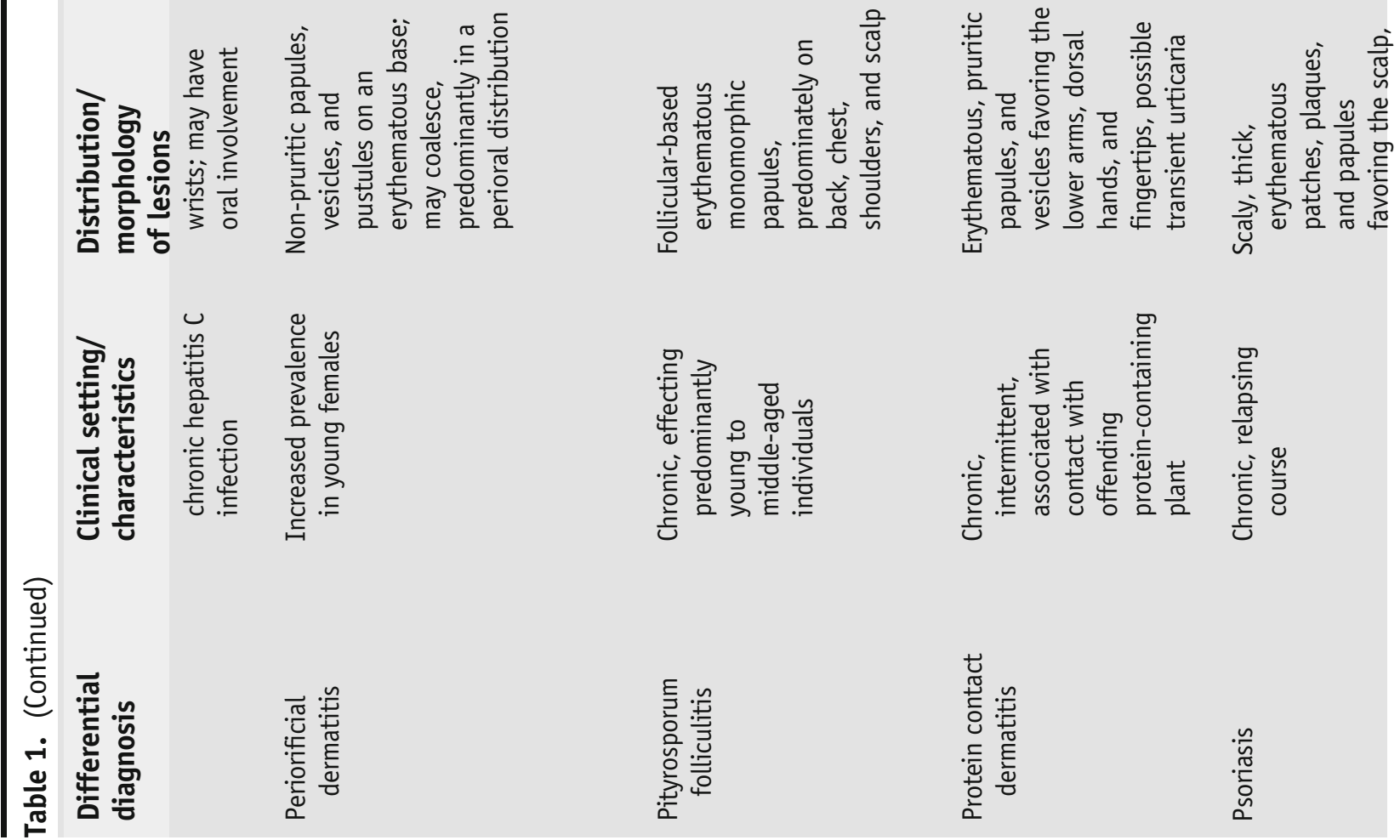


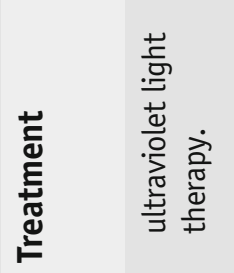

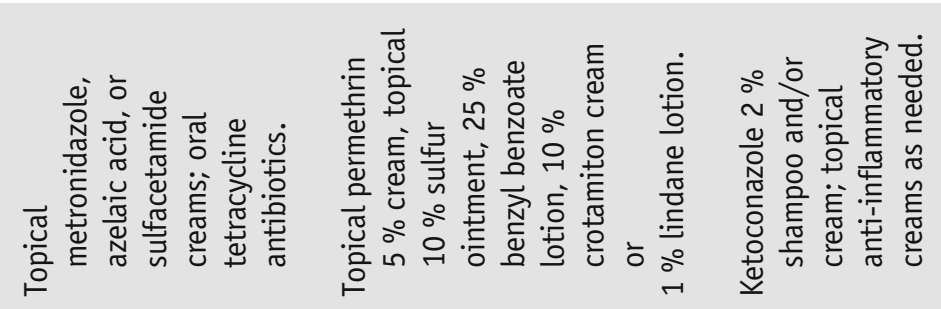

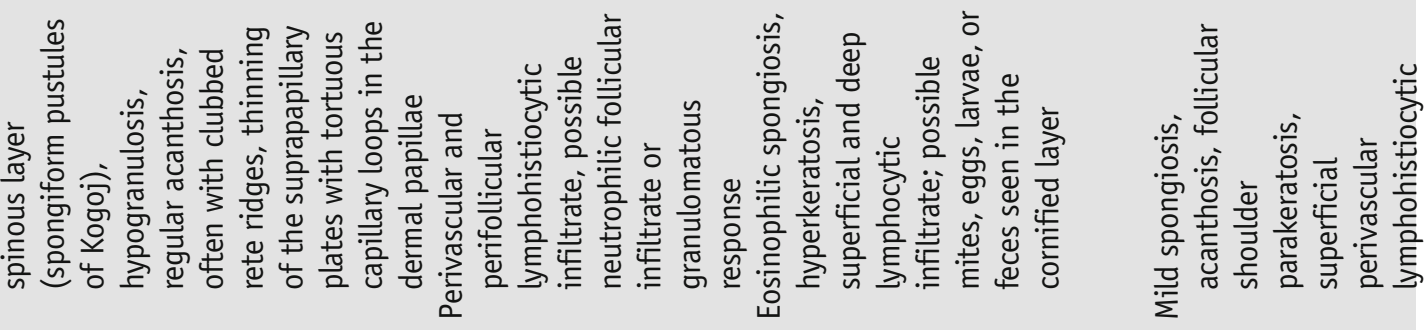

$\frac{5}{5}$
$\frac{5}{\bar{C}}$
$\frac{0}{0}$
$\frac{d}{\Sigma}$
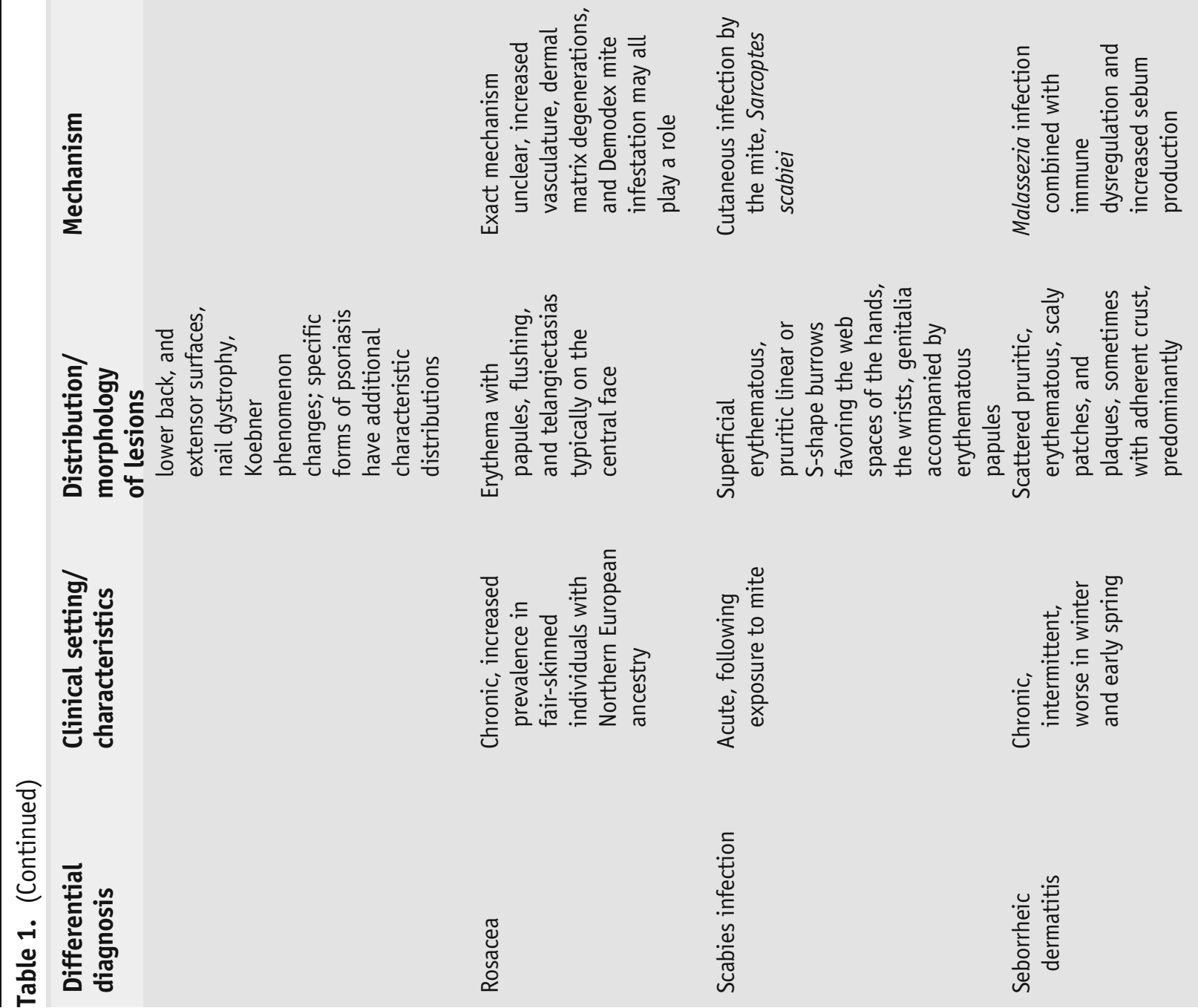


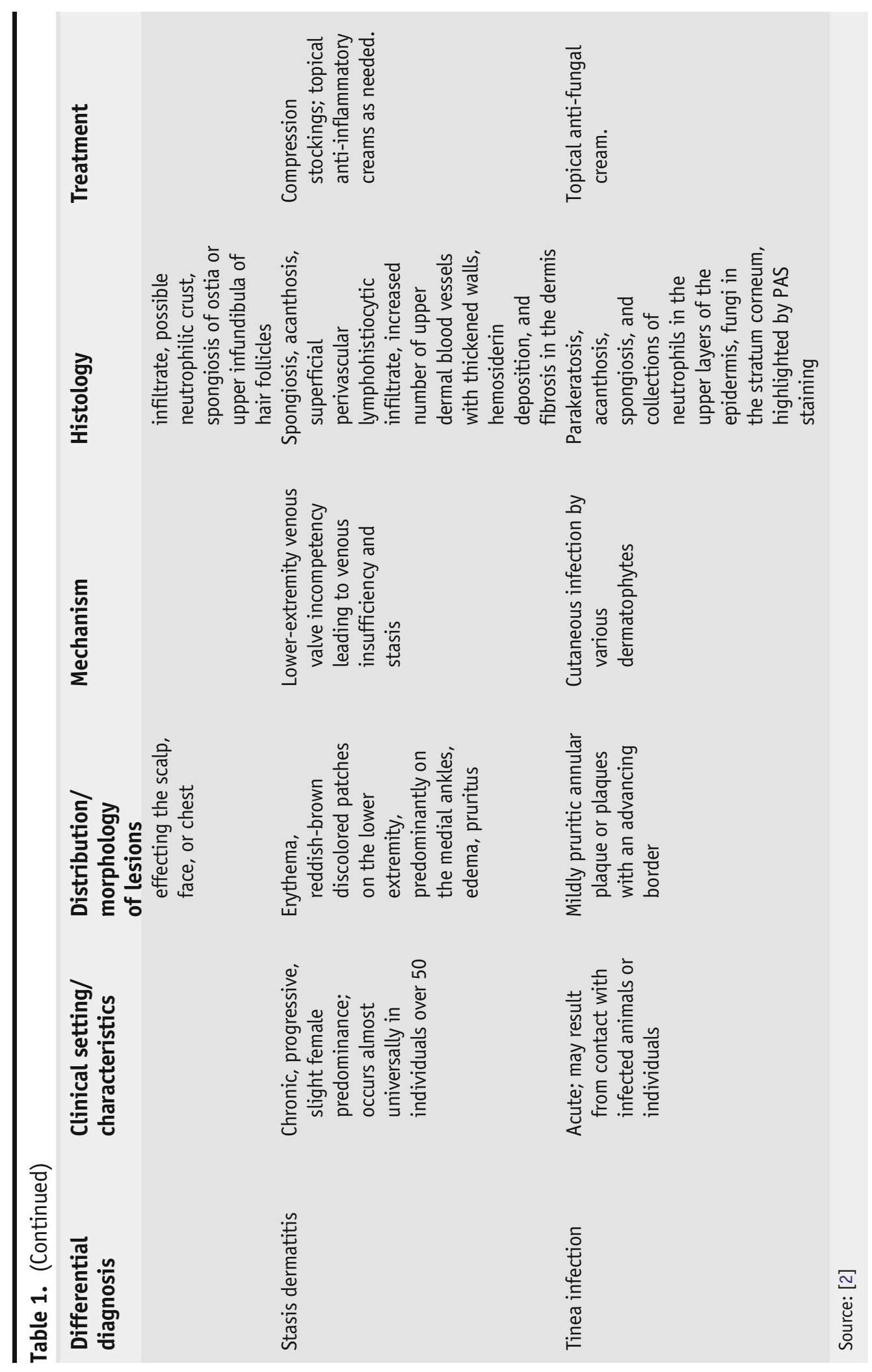


adjuvant management with topical and/or systemic treatment may be warranted to control disease activity $[4 \bullet]$. Of note, much of the working knowledge of the use of systemic immunosuppressants in severe refractory ACD is derived from the atopic dermatitis literature as well as the treatment of parthenium dermatitis, which is characteristically refractory in nature. In addition, allergen avoidance in patients with multifactorial dermatitis is unlikely to clear the other dermatitides without additional therapy.

\section{Antihistamines}

Antihistamines are generally safe and can be useful for relief of pruritus.

\section{Topical and systemic corticosteroids}

Evidence supports the continued use of topical corticosteroids as the mainstay of treatment of inflammatory, eczematous dermatitides, including ACD [5]. Long-term systemic corticosteroids use should be limited due to adverse effects. When possible, lower or taper the patient off of corticosteroids for patch testing, as they may suppress allergic patch test reactions [6].

\section{Topical and systemic calcineurin inhibitors}

Topical calcineurin inhibitors are effective options for topical steroidsparing treatment of ACD. Pimecrolimus $1 \%$ cream and tacrolimus 0.03 and $0.1 \%$ ointments have been shown to inhibit $\mathrm{T}$ lymphocyte and dendritic cell activation and thus work to inhibit both ICD and ACD [7]. Furthermore, pimecrolimus $1 \%$ cream, tacrolimus $0.1 \%$ ointment, clobetasol propionate $0.05 \%$ ointment, and triamcinolone acetonide $0.1 \%$ ointment were found to be equal in their ability to suppress experimentally induced nickel contact dermatitis in one study [8]. Pimecrolimus $1 \%$ cream and tacrolimus $0.1 \%$ ointment have excellent utility in treating ACD of the eyelids and genital skin. These topical calcineurin inhibitors also inhibit inflammatory cytokines; this is thought to explain the anti-pruritic effects as well as the burning sensation or pruritus reported by some patients when treatment is initiated [9]. Mixing the medicine with a bland refrigerated emollient can mitigate the burning sensation with initial application and can be then titrated up as tolerated.

Cyclosporine is a systemic calcineurin inhibitor that has been shown to be rapidly effective for the management of moderate to severe atopic dermatitis as well as severe generalized dermatitis needing to undergo evaluation with patch testing $[10,11 \bullet]$. It blocks activation of the c-Jun $\mathrm{N}$-terminal kinases and p38 signaling pathways that are involved in $\mathrm{T}$ cell activation following antigen recognition [12].

\section{Phototherapy (UVB, UVA)}

Narrowband UVB and UVA light therapies are effective in several inflammatory dermatologic conditions, including atopic dermatitis, psoriasis, and hand dermatitis. Contraindications to treatment include photosensitivity, photocontact dermatitis, photosensitive autoimmune disorders, the use of photosensitizing medications, and a history of non-melanoma skin cancer or melanoma. 
Azathioprine is a systemic immunosuppressive and anti-inflammatory agent useful for the treatment of dermatologic conditions with $\mathrm{T}$ and $\mathrm{B}$ cell over reactivity. It is considered a second-line option for moderate to severe atopic dermatitis [11•]. Thiopurine methyltransferase enzyme activity should be tested prior to initiation of treatment to determine correct dosing and identify individuals with susceptibility for side effects, notably pancytopenia.

\section{Methotrexate}

Methotrexate is a dihydrofolate reductase antagonist that provides immunosuppressive activity through inhibition of lymphocyte proliferation. Several trials demonstrate efficacy of methotrexate similar to azathioprine in the treatment of atopic dermatitis, with a significant improvement in several disease severity measures [13]. One small study of patients with parthenium dermatitis treated with methotrexate showed a decrease in disease severity after 1 month [14].

\section{Mycophenolate mofetil and mycophenolic acid}

Mycophenolate mofetil is a well-tolerated prodrug of the antimetabolite mycophenolic acid. Small studies have shown improvement in a majority of patients with refractory atopic dermatitis treated with mycophenolate mofetil [15].

Diet and lifestyle

Restrictive diets in sensitized patients' intake of nickel, balsam of Peru, and propylene glycol, have shown improved clinical outcome. The utility of diet modification in other cases of ACD remains controversial.

\section{Pediatric considerations}

ACD is as likely to occur in pediatric patients as it is in adults. The mainstay of treatment across all the ages is allergen avoidance. Notably, in the setting of atopic dermatitis, ACD is more likely to occur in children than adults.

\section{Compliance with Ethics Guidelines}

\section{Conflict of Interest}

Antoanella Calame declares that she has no conflict of interest.

Ashley Hamstra declares that she has no conflict of interest.

Jessica So declares that she has no conflict of interest.

Sharon E. Jacob served as an independent investigator on the safety and efficacy of T.R.U.E. Test ${ }^{\mathrm{TM}}$ (Smart Practice; Phoenix, AZ) panels 1.1, 2.1, and 3.1 in children and adolescents, Pediatric Research Equity Act (PREA-1) trial, and now serves as an investigator on PREA-2. She has served as a consultant for Johnson \& Johnson. She has no conflicts of interest associated with the specific subject matter in this manuscript. Carsten R Hamann is a first degree relative of Curt Hamann, owner of SmartPractice, a company that produces and sells diagnostic contact allergy products. 
Human and Animal Rights and Informed Consent

This article does not contain any studies with human or animal subjects performed by the author.

\section{References and Recommended Reading}

Papers of particular interest, published recently, have been

highlighted as:

- Of importance

1. Thyssen JP, Linneberg A, Menné T, Johansen JD. The epidemiology of contact allergy in the general population-prevalence and main findings. Contact Dermatitis. 2007;57(5):287-99. Review.

2. Calonje E, Brenn T, Lazar A, McKee P. McKee's pathology of the skin. 4th Ed. 2012. 185-193.

3. Jacob SE, Breithaupt AD. An important difference between "exposed" and "photodistributed" underscores the importance of identifying common reactions. J Clin Aesthet Dermatol. 2009;2(9):44-5.

4. $\quad$ Sheehan MP. Therapeutics in allergic contact dermatitis, when avoidance fails. Curr treatment options in allergy 2014 [published online 9/2014].

Excellent review of adjuvant therapies to consider when sufficient avoidance protocols yield suboptimal clinical outcomes

5. Saary J, Qureshi R, Palda V, DeKoven J, Pratt M, Skotnicki-Grant $S$, et al. A systematic review of contact dermatitis treatment and prevention. J Am Acad Dermatol. 2005;53(5):845-55.

6. Anveden I, Lindberg M, Andersen KE, et al. Oral prednisone suppresses allergic but not irritant patch test reactions in patients hypersensitive to nickel. Contact Dermatitis. 2004;50:298-303.

7. Lauerma AI, Stein BD, Homey B, et al. Topical FK 506: suppression of allergic and irritant contact dermatitis in the guinea pig. Arch Dermatol Res. 1994;286:337.

8. Bhardwaj SS, Jaimes JP, Liu A, et al. A double-blind randomized placebo-controlled pilot study comparing topical immunomodulating agents and corticosteroids for treatment of experimentally induced nickel contact dermatitis. Dermatitis. 2007;18:26.
9. Pereira U, Boulais N, Lebonvallet N, Pennec JP, Dorange G, Misery L. Mechanisms of the sensory effects of tacrolimus on the skin. Br J Dermatol. 2010;163(1):70-7.

10. Flori ML, Andreassi L. Patch tests after cyclosporin A treatment in hyperreactive patients. Contact Dermatitis. 1994;31(5):325.

11. Roekevisch E, Spuls PI, Kuester D, Limpens J, Schmitt J. Efficacy and safety of systemic treatments for moderateto-severe atopic dermatitis: a systematic review. J Allergy Clin Immunol. 2014;133(2):429-38.

A systemic review supporting the efficacy of short-term use of cyclosporine A and a review of the current efficacy and safety evidence for other systemic treatments.

12. Matsuda S, Moriguchi T, Koyasu S, Nishida E. T lymphocyte activation signals for interleukin-2 production involve activation of MKK6-p38 and MKK7-SAPK/JNK signaling pathways sensitive to cyclosporin A. J Biol Chem. 1998;273(20):12378-82.

13. Schram ME, Roekevisch E, Leeflang MM, et al. A randomized trial of methotrexate versus azathioprine for severe atopic eczema. J Allergy Clin Immunol. 2011;128:353-9.

14. Sharma V, Bhat R, Sethuraman G, Manchanda Y. Treatment of parthenium dermatitis with methotrexate. Contact Dermatitis. 2007;57(2):118-9.

15. Murray ML, Cohen JB. Mycophenolate mofetil therapy for moderate to severe atopic dermatitis. Clin Exp Dermatol. 2007;32:23-7. 